\title{
Longitudinal Analysis of the Relationship Between Changes in Smoking and Changes in Drinking in a Community Sample: The Winnipeg Health and Drinking Survey
}

\author{
Robert P. Murray \\ University of Manitoba \\ Robert A. Cribbie \\ University of Manitoba
}

\author{
Joseph A. Istvan \\ Oregon Health Sciences University \\ Gordon E. Barnes \\ University of Victoria
}

\begin{abstract}
Smoking cigarettes and drinking alcohol are positively correlated in cross-sectional studies of the general population. However, it is unclear whether changes in quantity of drinking over time are related to changes in amount of smoking over time. This investigation examined, with structural equation modeling, the relationship of changes in drinking to changes in smoking over 2 years among 344 adults who reported cigarette smoking and alcohol use at baseline in 1989-1990 or at follow-up in 1991-1992 or both. Surprisingly, no significant relationships were found between changes in smoking and changes in drinking. This lack of effect suggests that changes in the quantity or intensity of drinking and of smoking are not related in any important way in nonclinical populations.
\end{abstract}

Key words: smoking, drinking alcohol, community sample, behavior change, adults, longitudinal

During the past four decades, a multitude of clinical studies and epidemiologic investigations have examined the consequences of cigarette smoking and alcohol use for morbidity and mortality from a broad array of diseases. Less well understood, however, are the causes that contribute to the initiation of alcohol consumption or cigarette smoking or both and the factors associated with either the maintenance or the cessation of their use. Indeed, an understanding of factors associated with changes in tobacco and alcohol use may be particularly important because of the covariation commonly observed in their habitual consumption (Bobo, 1989; Hughes, 1995). A modest cross-sectional relationship between cigarette smoking and alcohol consumption has been widely reported in studies of the general population (Istvan \& Matarazzo, 1984; Revicki, Sobal, \& DeForge, 1991; Soeken \& Bausell, 1989; Zacny, 1990). In studies of persons with alcoholism in treatment, rates of smoking that are strikingly higher than those in the general

Robert P. Murray, Alcohol and Tobacco Research Unit, Department of Community Health Sciences, University of Manitoba, Winnipeg, Manitoba, Canada; Joseph A. Istvan, Department of Public Health and Preventive Medicine, Oregon Health Sciences University; Robert A. Cribbie, Department of Psychology, University of Manitoba; Gordon E. Barnes, School of Child and Youth Care, University of Victoria, Victoria, British Columbia, Canada.

Robert A. Cribbie is now at York University, Toronto, Ontario, Canada.

Funding for the Winnipeg Health and Drinking Survey and for this analysis was provided by the National Health Research and Development Program, Health Canada, Grants 6607-1474-DA and 6610-2166-101.

Correspondence concerning this article should be addressed to Robert P. Murray, Alcohol and Tobacco Research Unit, University of Manitoba, Room MS-750, 820 Sherbrook Street, Winnipeg, Manitoba R3A 1R9, Canada. E-mail: rmurray@hsc.mb.ca population have been found (Burling \& Ziff, 1988; DiFranza \& Guerrera, 1990), and cigarette smokers are about 1.3 times more likely to be drinkers than nonsmokers (Shiffman \& Balabanis, 1995). Although no study has examined the progression of alcohol use and cigarette smoking throughout adulthood in those who both smoke and drink, the array of cross-sectional investigations reporting an association between smoking and alcohol consumption in different age groups and across a range of consumption levels suggests that increases in the use of one with time likely will be paralleled by increases in the use of the other.

The general consistency of the relationship between alcohol use and cigarette smoking has suggested to many observers that there are common behavioral, pharmacologic, or genetic mechanisms mediating the relationship of cigarette use and tobacco use and that cigarette smoking or nicotine dependence may be a key factor in the maintenance of alcohol consumption in those with established patterns of alcohol use (Henningfield, Clayton, \& Polin, 1990; Hughes, 1993; Zacny, 1990). Indeed, it has been suggested that as the prevalence of smoking decreases in all but the most nicotine dependent, the population of cigarette smokers largely consists of those with alcohol use problems (Hughes, 1995).

To date, the relationship of changes in smoking status to subsequent changes in alcohol intake has been investigated largely in clinic-based studies. The results of these studies generally suggest that persons who have alcoholism and who have quit smoking prior to alcoholism treatment are no less likely to successfully complete treatment than those who continue to smoke (e.g., Abrams et al., 1992; Joseph, Nichol, \& Anderson, 1993) and may even be marginally more successful (Bobo, Gilchrist, Schilling, Noach, \& Schinke, 1987; Miller, Hedrick, \& Taylor, 1983). However, inasmuch as the participants in these studies all had alcoholism and either a current or a past history of involvement with 
clinic-based alcoholism treatment, these findings may not be readily generalizable to the broader population of problem drinkers who stop smoking.

Few epidemiologic investigations have examined the effect of changes in smoking on changes in drinking. For instance, Gordon and Doyle (1986), studying a group of over 1,700 men longitudinally for 18 years, found that despite high correlations between smoking and drinking, there were no significant correlations between changes in drinking and changes in smoking habits. On the other hand, in a study of World War II-era veteran twins, smoking cessation was associated with apparent increases in alcohol intake over a 16-year period (Carmelli, Swan, \& Robinette, 1993). In a retrospective survey of young adults in Detroit, Breslau, Peterson, Schultz, Andreski, and Chilcoat (1996) found no differences in the likelihood of remission of alcohol use or alcohol dependence in persons who had alcoholism and who stopped smoking. In a study of risk factors for heart disease, Nothwehr, Lando, and Bobo (1995) reported data from a cohort of 3,643 adults who resided in three intervention or three control communities. No differences were found in mean alcohol intake between those who quit smoking and those who did not. Also, alcohol intake at baseline was not related to smoking status at follow-up. Murray, Istvan, and Voelker (1996) examined the alcohol use of 5,887 adult smokers in a randomized clinical trial in which there was a smoking cessation intervention. After 1 year in the study, those assigned to the smoking cessation intervention did not differ in their use of alcohol from those assigned to usual care, although there was a highly significant difference between these groups in the use of cigarettes.

Studies examining the effects of alcohol on nicotine consumption are somewhat more plentiful than those examining nicotine and its effect on alcohol use. Not surprisingly, persons who have alcoholism and who are currently drinking and smoking are less likely than those who do not have alcoholism but who smoke to quit smoking (e.g., Burling \& Ziff, 1988; DiFranza \& Guerrera, 1990). However, persons recovering from alcoholism are commonly interested in quitting smoking (Bobo, McIlvain, Gilchrist, \& Bowman, 1996), and among those who enter clinical interventions to do so, rates of success are generally no lower than those of persons who do not have alcoholism but who smoke (Covey, Glassman, Stetner, \& Becker, 1993; Hurt et al., 1995). However, a brief smoking cessation intervention shortly after completion of alcoholism treatment may not be effective (Centers for Disease Control and Prevention, 1997).

Survey data examining the cessation of smoking in those with a history of alcohol use problems generally show that a history of alcohol use problems does not impair smoking cessation. For example, DeSoto, O'Donnell, and DeSoto (1989) reported a lifetime rate of smoking cessation in persons recovering from alcoholism $(53 \%)$ that is probably indistinguishable from that of all other smokers, whereas Sobell, Sobell, and Toneatto (1992) found that $67 \%$ of a group of persons recovering from alcoholism had successfully quit smoking. Breslau et al. (1996) also found that smokers with remittent alcoholism were no less likely to quit smoking than were those without a history of alcoholism. In one study that provided contrasting results, an analysis of Epidemiologic Catchment Area data from North Carolina found that a lifetime history of alcohol dependence was associated with a lower rate of smoking cessation (Covey, Hughes, Glassman, Blazer, \& George, 1994).

Although the majority of those who smoke do use alcohol, only a minority of smokers who drink are currently alcohol dependent (13 to 15\%) (Hughes, 1995). However, alcohol use often seems to be involved in situations in which a relapse to smoking occurs among those who have recently quit smoking (Baer \& Lichtenstein, 1988). For example, Murray, Istvan, Voelker, Rigdon, and Wallace (1995) found that alcohol use affected success in a smoking cessation intervention among 3,977 men and women. Among both men and women, binge drinking (eight or more drinks at a sitting) but not drinking volume was related to smoking status after 1 year.

In summary, although most literature suggests that levels of alcohol use are related to levels of smoking, the majority of studies have examined persons involved in some form of clinical intervention. Population-based studies, in contrast, have tended to be more inconclusive. The purposes of this study were (a) to examine the relationship between smoking and drinking alcohol in a community sample, distinguishing between genders and categories of age, and (b) to assess the evidence that changes in smoking over time are related to changes in drinking or that changes in drinking over time are related to changes in smoking by use of a modeling approach that permits simultaneous control of a complex set of interrelationships.

\section{Method}

\section{Sample}

The Winnipeg Health and Drinking Survey was a general-population, longitudinal survey that examined alcohol and nicotine consumption, consequences of consumption, and a wide range of variables speculated to be related to or causes of use. Participants were interviewed in two waves, approximately 2 years apart (1989-1990 and 1991-1992).

Wave 1. The original sample was a random list of 4,000 names provided by the universal medicare administration, the Manitoba Health Services Commission. In the course of interviewing, we randomly subsampled 2,753 names from this list and mailed introductory letters to these potential participants. No payment was offered. An attempt to contact respondents by phone followed the letter by 1 to 3 weeks. An average of five attempts were made to contact each individual (range of 3 to 11 calls). In total, 446 of the 2,753 could not be found; 336 were ineligible because they had moved away $(n=166)$, they had insufficient command of the English language $(n=155)$, or they were currently institutionalized or had died $(n=15)$; and 704 refused to participate. Ten were lost because of administrative error. The result was that 615 male and 642 female respondents completed Wave 1 interviews. The response rate, defined as the ratio of completed interviews to the number of persons who were found and eligible, was $64.1 \%$. The Wave 1 interviews were conducted in the participant's home or occasionally in another location preferred by the participant. The participants read and signed a consent form before the interview began. For additional information regarding sampling techniques, measures, and questionnaire procedures, see Murray, Barnes, and Patton (1994) and Barnes, Murray, Patton, Bentler, and Anderson (2000).

Wave 2. At 1.5 years after the date of the Wave 1 interview, participants were contacted by phone to confirm their current address and to remind them of the Wave 2 interview, which was to occur in about 6 months. Six months later, within a window of 23 to 25 months after the date of the Wave 1 interview, each participant was again contacted by phone to arrange a date and time for the Wave 2 interview. Of the 1,257 interviewed at Wave 1, 57 could not be located for Wave 2, 4 had since 
died, 4 were institutionalized, 83 had moved away, and 121 refused to complete Wave 2. In total, 988 participants completed both Wave 1 and Wave 2 interviews. Wave 1 participants who responded to Wave 2 did not differ from those who did not respond in their overall mean (standard deviation) daily use of cigarettes smoked per day, 4.70 (9.24) and 5.05 (9.81), respectively, $t(1255)=0.552, p=.58$. Respondents and nonrespondents to Wave 2 did not differ in age, gender, marital status, ounces of ethanol consumed per day, the use of more than eight drinks per occasion, or reports of being drunk more than 1 day in a row (Barnes et al., 2000, chapter 3). They did differ in univariate analysis on the sum of problems attributable to alcohol. However, in logistic regression models done separately for men and women, respondents and nonrespondents to Wave 2 were not differentiated by alcohol variables (use, dependence, or consequences) when the models were adjusted by demographic characteristics (Barnes et al., 2000, chapter 3). The analysis reported below was based on 344 individuals who reported at least some smoking and some drinking, at least during one wave of the study.

\section{Measures}

After the qualifying question, "Do you now smoke cigarettes?" participants were asked, "On the average, how many cigarettes do you now smoke per day?" They were also asked, "How often do you usually have wine?" The response choices ranged from "Three or more times a day" to "I have never had wine." Next they were asked, "Think of all the times you have had wine recently. When you drink wine, how many glasses do you usually have?" The response choices were one or two glasses, three or four glasses, five or six glasses, or more than six glasses. The product of the responses to these two questions was expressed as the number of drinks of wine per day. Similar pairs of questions were asked about beer and liquor. The volume of alcohol used in this study is the sum of the estimates for wine, beer, and liquor expressed as number of drinks per day. The number of times a person was drunk was based on answers to the question, "About how often do you drink enough to get high or tight, on the average?"

\section{Statistical Methods}

The principal data analyses used structural equation modeling (SEM). The model used in the current study was based on research by Raykov (1993, 1994, 1997), MacCallum, Kim, Malarkey, and Kiecolt-Glaser (1997), Duncan et al. (1997), and others for measuring change and determining correlates of change (Figure 1). In this study, the interest was in determining whether changes in alcohol consumption would be correlated with changes in cigarette smoking. A measure of alcohol consumption change was established with the use of a latent change variable, estimated from changes in overall consumption of alcohol and in the number of times per month an individual reported having been drunk. A measure of cigarette smoking change was computed by subtracting the number of cigarettes smoked at Wave 1 from the number of cigarettes reported at Wave 2. A latent measure of cigarette smoking change was established from the single covariate (cigarette smoking change) by setting the path coefficient equal to one and the variance of the latent measure equal to zero. The relationship of interest in this study is that between the latent measures of alcohol change and smoking change. Cribbie and Jamieson (2000) found SEM to be a reliable estimator of change in variables measured at two time points, allowing for estimation of the relationships between latent measures of change.

The EQS software package (Bentler, 1995) was used for all SEM analyses, with maximum-likelihood estimation. The fit of the structural model to the data was evaluated with the goodness-of-fit index (GFI) (Joreskog \& Sorbom, 1989) and the comparative fit index (CFI) (Bentler, 1990). Fan, Thompson, and Wang (1999) reported several advantages of each of the selected indices for evaluating the fit of a model to sample data. Specifically, the GFI is sensitive to model misspecifications and is not

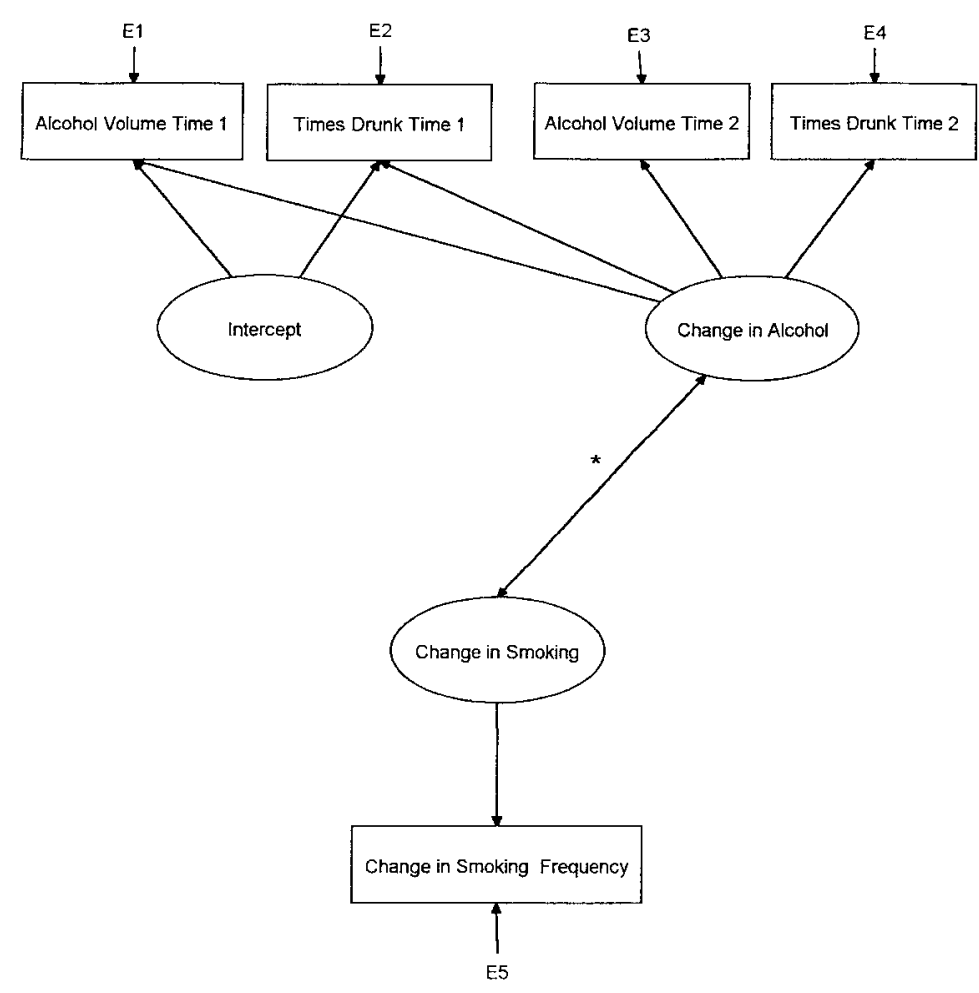

Figure 1. Hypothetical model of the relationships between changes in drinking and changes in smoking. E1-E5 represent error terms associated with the observed variables. The asterisk indicates the path to be estimated. 
overly influenced by the type of estimation procedure selected, whereas the CFI is relatively insensitive to the sample size. To avoid capitalizing on chance, no data-driven model modifications were performed. Although the analyses were correlational in nature, the longitudinal design of the study permitted strong recommendations about the causal nature of the relationships.

With the limited number of variables in our models, the use of continuous rather than dichotomous variables provided more true variability and hence more power. Specifically, although the consumption of eight or more drinks at a sitting (binge drinking) and smoking status (yes or no) are commonly used, the use of the number of times drunk and amount of smoking was preferred in these analyses.

\section{Results}

Among the 344 people included in these analyses, the mean (standard deviation) age of the women was 37.9 (12.4) and that of the men was $38.9(12.1), 10.1 \%$ of women and $8.4 \%$ of men were unemployed, $53.9 \%$ of women and $63.9 \%$ of men had an annual family income of $\$ 35,000$ (Canadian) or more, $66.3 \%$ of men and $59.6 \%$ of women were married, and women had a mean (standard deviation) of 12.4 (2.4) years of education compared to 12.6 (2.4) years for men.

Descriptive data for each of the alcohol and smoking variables used in the study are presented in Table 1 . As commonly observed elsewhere, young and middle-aged men consumed the greatest volume of alcohol at baseline and older women consumed the least. These relationships were preserved across Wave 1 and
Wave 2, except for older men's drinking volume, which was unusually high because of the presence of a single outlier included in the analysis. The reporting of number of times drunk per month increased in Wave 2 among men and older women. The mean number of cigarettes smoked per day reported by all participants in the analyses was more consistent between age and gender cells.

There were 38 participants in the Wave 2 sample who stopped smoking in the 2 years between Wave 1 and Wave 2 and 26 individuals who reported smoking only at Wave 2. Among those who reported being drunk at least once in the past year, there were 133 who reported this only at Wave 1 and 54 who reported it only at Wave 2.

In the traditional approach, the group means for number of drinks of alcohol per day were compared between those who quit smoking between Wave 1 and Wave 2 ( $n=32$ with complete data) and those who did not $(n=839)$. These means were 0.871 and $0.871, t(869)=-0.002, p=.999$.

The correlations, combined sample means, and standard deviations for the observed variables in the structural model are presented in Table 2. The simple correlation between change in drinks consumed per day between Wave 1 and Wave 2 and change in cigarettes smoked per day was $.008, p=.91$.

The initial factor model (Figure 1) fit the data well, GFI $=.967$, CFI $=.956, \chi^{2}(3, N=250)=31.228, p<.01$. Each of the observed alcohol measures contributed significantly to the latent measure of alcohol change: number of drinks per day, $z=9.636$,

Table 1

Responses for Men and Women in Each Age Category on the Variables Used in the Latent Variable Model (Wave 1 and Wave 2)

\begin{tabular}{|c|c|c|c|c|c|c|}
\hline \multirow[b]{2}{*}{ Behavioral measure } & \multicolumn{3}{|c|}{ Men } & \multicolumn{3}{|c|}{ Women } \\
\hline & $\begin{array}{c}18-30 \\
(68)\end{array}$ & $\begin{array}{c}31-49 \\
(61)\end{array}$ & $\begin{array}{c}50-65 \\
(37)\end{array}$ & $\begin{array}{c}18-30 \\
(83)\end{array}$ & $\begin{array}{c}31-49 \\
(59)\end{array}$ & $\begin{array}{c}50-65 \\
(36)\end{array}$ \\
\hline \multicolumn{7}{|c|}{ Number of drinks consumed per day } \\
\hline \multicolumn{7}{|l|}{ Wave 1} \\
\hline$M$ & 1.72 & 1.88 & 1.17 & 1.15 & 0.87 & 0.52 \\
\hline$S D$ & 1.64 & 3.86 & 1.30 & 2.72 & 3.40 & 0.62 \\
\hline \multicolumn{7}{|l|}{ Wave 2} \\
\hline$M$ & 1.56 & 0.81 & 2.25 & 0.96 & 0.45 & 0.59 \\
\hline$S D$ & 1.66 & 0.91 & 4.30 & 1.64 & 0.52 & 0.86 \\
\hline
\end{tabular}

Number of times drunk per month

\begin{tabular}{ccccccc} 
Wave 1 & & & & & & \\
$M$ & 1.67 & 1.54 & 0.46 & 1.00 & 1.01 & 0.36 \\
$S D$ & 2.04 & 4.34 & 1.06 & 1.65 & 4.00 & 1.10 \\
Wave 2 & & & & & & \\
$M$ & 2.09 & 1.46 & 1.58 & 1.02 & 0.22 & 1.48 \\
$S D$ & 3.16 & 5.06 & 5.85 & 2.59 & 0.44 & 5.98 \\
\hline
\end{tabular}

Number of cigarettes smoked per day

\begin{tabular}{ccccccc} 
Wave 1 & & & & & & \\
$M$ & 15.3 & 19.4 & 20.1 & 15.0 & 18.1 & 15.0 \\
$S D$ & 9.95 & 11.4 & 11.5 & 8.86 & 10.6 & 7.57 \\
Wave 2 & & & & & & \\
$M$ & 16.6 & 17.7 & 18.0 & 14.5 & 16.3 & 14.3 \\
$S D$ & 8.91 & 10.1 & 9.75 & 9.10 & 8.74 & 7.86 \\
\hline
\end{tabular}

Note. Participants were grouped by gender and by age in years; values in parentheses indicate number of participants per group. 
Table 2

Correlations, Means, and Standard Deviations for Each of the Variables Used in the Latent Variable Model $(N=344)$

\begin{tabular}{lrrrrr}
\hline & \multicolumn{5}{c}{ Result for variable } \\
\cline { 2 - 6 } Variable & 1 & 2 & 3 & 4 & 5 \\
\hline 1 & - & & & & \\
2 & .395 & - & & & \\
3 & .821 & .331 & - & & \\
4 & .364 & .561 & .345 & -.004 & - \\
5 & -.108 & -.077 & -.065 & -.004 \\
$M$ & 1.278 & 1.054 & 1.103 & 1.263 & -1.050 \\
$S D$ & 2.694 & 1.886 & 2.812 & 3.834 & 7.341 \\
\hline
\end{tabular}

Note. Variables: 1 and 2, numbers of drinks per day in Wave 1 and Wave 2, respectively; 3 and 4, numbers of times drunk per month in Wave 1 and Wave 2, respectively; 5, change in smoking between Wave 1 and Wave 2. Results are correlations, unless otherwise indicated.

$p<.001$, and number of times drunk, $z=18.234, p<.001$. However, the relationship between the latent measure of alcohol change and the latent measure of smoking change was not significant, $z=-.075, p>.05$. In order to determine whether removing the path between the latent measures of alcohol change and smoking change would significantly decrease the overall fit of the model, a likelihood-ratio test was used to compare the original model (with the path included) to the modified model, hypothesizing a null relationship between alcohol change and smoking change. Hancock and Lawrence (1998) found likelihood-ratio tests (often referred to as chi-square difference tests) to be the most reliable methods for evaluating the significance of individual parameters in SEM compared with various model modification strategies (e.g., Wald test and Lagrange multiplier). The modified model also fit the data well, GFI $=.966, \mathrm{CFI}=.957, \chi^{2}(4, N=$ $250)=30.942, p<.01$. The chi-square difference test $(31.228-30.942)$ was not significant, $\chi^{2}(1)=.286, p>.05$, indicating that adding the path between the latent measures of alcohol change and smoking change did not significantly improve the fit of the model and confirming a lack of association between changes in alcohol behavior and changes in smoking behavior.

Further explorations of the data included evaluating the relationship between changes in smoking and drinking behaviors for specific subgroups of the sample. No significant relationship was found between changes in smoking behavior and changes in drinking behavior for men, women, younger participants (18 to 34 years old), middle-aged participants (35 to 49 ), or older participants (50 to 65$)$.

Although the sample sizes for the subgroup analyses were small (e.g., $n=73$ for 50- to 65-year-old participants), results from Boomsma (1985) and Gerbing and Anderson (1985) suggest that these sample sizes may be sufficient for detecting relationships in a model with only 12 parameters to be estimated. Further, the correlation between changes in drinking and changes in smoking never exceeded .05 for any of the subgroups.

\section{Discussion}

Although it seems to be generally presumed that there is a causal relationship between drinking alcohol and smoking cigarettes, there have been relatively few investigations examining whether changes in drinking are related to changes in smoking or whether changes in smoking are related to changes in drinking. The results of this study fail to confirm the presence of a relationship between changes in drinking and changes in smoking in the general adult population.

The relationship between changes in smoking and changes in drinking in this study was remarkably weak. The mean numbers of drinks consumed per day at Wave 2 were identical for those who had quit smoking and those who had not. The overall correlation between changes in smoking and changes in drinking was less than .01. Interest in this issue has been driven by concerns about the likely success of intervening with smoking among individuals who are heavy drinkers and, for that matter, the likely success in the treatment of drinking among those who continue to smoke. In the adult population at large, there appears to be little empirical basis for such concerns. Further, analysis by gender or by age group, although weakened by smaller sample sizes, was still adequate in power on the basis of conventional criteria, and the relationship was weak in each instance.

We should also note that hospitalization or other illness events may be the single most important correlate of changes in smoking or drinking habits in community-based samples. We did not control for or eliminate respondents with illness or hospitalization, a step that likely would have biased the analyses toward rejection of the null hypothesis. Nonetheless, no longitudinal association of changes in drinking with changes in smoking was observed.

It should also be acknowledged that the majority of prior research has focused on the impact of clinical intervention for alcohol abstention on changes in smoking behavior or, conversely, the effect of clinical smoking cessation intervention on changes in alcohol use. Our findings thus may not be readily generalizable to clinical settings or to individuals with diagnosed tobacco or alcohol dependence. However, because the vast majority of individuals who modify their drinking or smoking habits do so without formal clinical intervention (see, for example, Sobell, Cunningham, \& Sobell, 1996), the natural covariation of smoking and drinking habits merits continued investigation.

It is important to recognize that factors influencing situational or short-term temporal covariation in smoking and drinking — such as common environmental cues or pharmacologic interactions-may be quite different from those affecting long-term patterns of use. Thus, individual differences in covariation of alcohol and tobacco use over longer periods of time are influenced by broader changes in access and availability, social approval or disapproval, changes in excise taxation or net cost, or local regulations regarding public use. It also seems probable that such long-term societal and normative influences will affect use of alcohol and tobacco differentially, reducing their joint covariation over time. Unfortunately, changes in public policy and other broader social influences are often given no more than passing acknowledgment by behavioral scientists as initiators of change in individual behavior.

This study constituted a relatively suitable design for the question that was addressed. It may have generated more confidence with a larger sample, and such a replication is no doubt worth conducting. A larger sample and hence more power also may have allowed the exploration of more usual expressions of substance use, such as the dichotomous use of cigarettes (smoking versus not 
smoking) and the use of eight or more drinks at a sitting as an estimate of binge drinking.

How does this investigation contribute to an understanding of the relationship between alcohol use and tobacco use? In the general adult population, in which the majority of alcohol users are not physically dependent and in which the majority of tobacco users more likely are dependent, there appears to be little evidence that changes in alcohol use are related to changes in tobacco use. One of the oldest axioms in statistics is that the null hypothesis cannot be proven. Thus, these findings should not be regarded as clearly definitive. Nonetheless, we believe that a conservative and appropriate conclusion is that relatively long-term changes in the use of alcohol and the use of tobacco by individuals in the general population may occur in a manner independent of each other.

\section{References}

Abrams, D. B., Rohsenow, D. J., Niaura, R. S., Pedraza, M., Longabaugh, R., Beattie, M. C., Binkoff, J. A., Noel, N. E., \& Monti, P. M. (1992). Smoking and treatment outcome for alcoholics: Effects on coping skills, urge to drink, and drinking rates. Behavior Therapy, 23, 283-297.

Baer, J., \& Lichtenstein, E. (1988). Classification and prediction of smoking relapse episodes: An exploration of individual differences. Journal of Consulting and Clinical Psychology, 56, 104-110.

Barnes, G. E., Murray, R. P., Patton, D., Bentler, P. M., \& Anderson, R. E. (2000). The addiction-prone personality. New York: Kluwer Academic/ Plenum.

Bentler, P. M. (1990). Comparative fit indexes in structural models. Psychological Bulletin, 107, 238-246.

Bentler, P. M. (1995). EQS structural equations program manual. Encino, CA: Multivariate Software.

Bobo, J. K. (1989) Nicotine dependence and alcoholism epidemiology and treatment. Journal of Psychoactive Drugs, 21, 323-329.

Bobo, J. K., Gilchrist, L. D., Schilling, R. F., Noach, B., \& Schinke, S. P. (1987). Cigarette smoking cessation attempts by recovering alcoholics. Addictive Behaviors, 12, 209-216.

Bobo, J. K., McIlvain, H. E., Gilchrist, L. D., \& Bowman, A. (1996). Nicotine dependence and intentions to quit smoking in three samples of male and female recovering alcoholics and problem drinkers. Substance Use and Misuse, 31, 17-33.

Boomsma, A. (1985). Nonconvergence, improper solutions, and starting values in LISREL maximum likelihood estimation. Psychometrika, 50, 229-242.

Breslau, N., Peterson, E., Schultz, L., Andreski, P., \& Chilcoat, H. (1996). Are smokers with alcohol disorders less likely to quit? American Journal of Public Health, 86, 985-990.

Burling, T. A., \& Ziff, D. C. (1988). Tobacco smoking: A comparison between alcohol and drug abuse inpatients. Addictive Behaviors, 13, 185-190.

Carmelli, D., Swan, G. E., \& Robinette, D. (1993). The relationship between quitting smoking and changes in drinking in World War II veteran twins. Journal of Substance Abuse, 5, 103-116.

Centers for Disease Control and Prevention. (1997). Efforts to quit smoking among persons with a history of alcohol problems-Iowa, Kansas, and Nebraska, 1995-96. Morbidity and Mortality Weekly Reports, 46, $1144-1148$

Covey, L. S., Glassman, A. H., Stetner, F., \& Becker, J. (1993). Effect of history of alcoholism or major depression on smoking cessation. American Journal of Psychiatry, 150, 1546-1547.

Covey, L. S., Hughes, D. C., Glassman, A. H., Blazer, D. G., \& George, L. K. (1994). Ever-smoking, quitting, and psychiatric disorders: Evidence from the Durham, North Carolina, Epidemiologic Catchment Area. Tobacco Control, 3, 222-227.
Cribbie, R. A., \& Jamieson, J. L. (2000). Structural equation models and the regression bias for measuring change. Educational and Psychological Measurement, 60, 893-907.

DeSoto, C. B., O’Donnell, W. E., \& DeSoto, J. L. (1989). Long-term recovery in alcoholics. Alcoholism: Clinical and Experimental Research, 13, 693-697.

DiFranza, J. R., \& Guerrera, M. P. (1990). Alcoholism and smoking. Journal of Studies on Alcohol, 51, 130-135.

Duncan, T. E., Duncan, S. C., Alpert, A., Hops, H., Stoolmiller, M., \& Muthen, B. (1997). Latent variable modeling of longitudinal and multilevel substance abuse data. Multivariate Behavioral Research, 32, $275-318$.

Fan, X., Thompson, B., \& Wang, L. (1999). Effects of sample size, estimation methods and model specification on structural equation modeling fit indexes. Structural Equation Modeling, 6, 56-83.

Gerbing, D. W., \& Anderson, J. C. (1985). The effects of sampling error and model characteristics on parameter estimation for maximum likelihood confirmatory factor analysis. Multivariate Behavioral Research, 20, 255-271.

Gordon, T., \& Doyle, J. T. (1986). Alcohol consumption and its relationship to smoking, weight, blood pressure, and blood lipids. Archives of Internal Medicine, 146, 262-265.

Hancock, G. R., \& Lawrence, F. R. (1998, April). Finite sample behavior of the likelihood ratio, Wald, and Lagrange multiplier tests: Bias and variability in univariate noncentrality parameter estimation. Paper presented at the 1998 annual meeting of the American Educational Research Association, San Diego, CA.

Henningfield, J. E., Clayton, R., \& Polin, W. (1990). Involvement of tobacco in alcoholism and illicit drug use. British Journal of Addiction, 85, 279-292.

Hughes, J. R. (1993). Treatment of smoking cessation in smokers with past alcohol/drug problems. Journal of Substance Abuse Treatment, 10, 181187.

Hughes, J. R. (1995). Clinical implications of the association between smoking and alcoholism. In J. B. Fertig \& J. P. Allen (Eds.), Alcohol and tobacco: From basic science to clinical practice (NIDA Research Monograph 30, NIH Publication No. 95-3931, pp. 171-185). Washington, DC: U.S. Government Printing Office.

Hurt, R. D., Dale, L. C., Offord, K. P., Croghan, I. T., Hayes, J. T., \& Gomez-Dahl, L. (1995). Nicotine patch therapy for smoking cessation in recovering alcoholics. Addiction, 90, 1541-1546.

Istvan, J., \& Matarazzo, J. D. (1984). Tobacco, alcohol, and caffeine use: Review of their relationships. Psychological Bulletin, 95, 301-326.

Joreskog, K., \& Sorbom, D. (1989). LISREL 7: A guide to the program and applications (2nd ed.). Chicago: SPSS.

Joseph, A. M., Nichol, K. L., \& Anderson, H. (1993). Effect of treatment for nicotine dependence on alcohol and drug treatment outcomes. Addictive Behaviors, 18, 635-644.

MacCallum, R. C., Kim, C., Malarkey, W. B., \& Kiecolt-Glaser, J. K. (1997). Studying multivariate change using multilevel models and latent curve models. Multivariate Behavioral Research, 32, 215-253.

Miller, W. R., Hedrick, K. E., \& Taylor, C. A. (1983). Addictive behaviors and life problems before and after behavioral treatment of problem drinkers. Addictive Behaviors, 8, 403-412.

Murray, R. P., Barnes, G. E., \& Patton, D. (1994). The relative performance of diverse measures of alcohol abuse and dependence in a community sample. Journal of Studies on Alcohol, 55, 72-80.

Murray, R. P., Istvan, J. A., \& Voelker, H. T. (1996). Does cessation of smoking cause a change in alcohol consumption? Evidence from the Lung Health Study. Substance Use and Misuse, 31, 141-156.

Murray, R. P., Istvan, J. A., Voelker, H. T., Rigdon, M., \& Wallace, M. D. (1995). Level of involvement with alcohol and success at smoking cessation in the Lung Health Study. Journal of Studies on Alcohol, 56, $74-82$. 
Nothwehr, F., Lando, H. A., \& Bobo, J. K. (1995). Alcohol and tobacco use in the Minnesota Heart Health Program. Addictive Behaviors, 20, 463470.

Raykov, T. (1993). A structural equation model for measuring residualized true change and discerning patterns of growth or decline. Applied Psychological Measurement, 17, 53-71.

Raykov, T. (1994). Studying correlates and predictors of longitudinal change using structural equation modeling. Applied Psychological Measurement, 18, 63-77.

Raykov, T. (1997). Simultaneous study of individual and group patterns of latent longitudinal change using structural equation modeling. Structural Equation Modeling, 4, 212-236.

Revicki, D., Sobal, J., \& DeForge, B. (1991). Smoking status and the practice of other unhealthy behaviors. Family Medicine, 23, 361-364.

Shiffman, S., \& Balabanis, M. (1995). Association between alcohol and tobacco. In J. B. Fertig \& J. P. Allen (Eds.), Alcohol and tobacco: From basic science to clinical practice (NIDA Research Monograph 30, NIH Publication No. 95-3931, pp. 17-36). Washington, DC: U.S. Government Printing Office.

Sobell, L. C., Cunningham, J. A., \& Sobell, M. B. (1996). Recovery from alcohol problems with and without treatment: prevalence in two population surveys. American Journal of Public Health, 86, 966972.

Sobell, L. C., Sobell, M. B., \& Toneatto, T. (1992). Recovery from alcohol problems without treatment. In N. Heather, W. R. Miller, and J. Greeley (Eds.), Self-control and the addictive behaviors (pp. 198-242). New York: Maxwell MacMillan.

Soeken, K. L., \& Bausell, R. B. (1989). Alcohol use and its relationship to other addictive and preventive behaviors. Addictive Behaviors, 14, 459464.

Zacny, J. P. (1990). Behavioral aspects of alcohol-tobacco interactions. Recent Developments in Alcoholism, 8, 205-219. 